\title{
STABLE AND ISOPERIMETRIC REGIONS IN ROTATIONALLY SYMMETRIC TORI WITH DECREASING GAUSS CURVATURE
}

\author{
ANTONIO CAÑETE
}

\begin{abstract}
In this work we classify the stable regions (second order minima of perimeter under an area constraint) in tori of revolution with piecewise continuous decreasing Gauss curvature from the longest parallel and with a horizontal symmetry. Some applications to isoperimetric problems are also given.
\end{abstract}

\section{INTRODUCTION}

In a Riemannian surface $M$ we may consider the isoperimetric problem consisting of finding the least perimeter sets in $M$ enclosing a given area $a_{0}$, with $a_{0} \leqslant \operatorname{area}(M)$. If such a set exists, then it is called an isoperimetric region. In the last years this problem has been of great interest, but only for certain surfaces isoperimetric regions have been completely classified (see $\mathbf{B C},[\mathbf{P}, \mathbf{T},[\mathbf{H H M 1},[\mathbf{H H M 2},[\mathbf{R})$ ). A very important related concept is the one of stability: a stable region is a second order minimum of perimeter under any variation preserving the area enclosed. Variational formulae for perimeter and area imply that the boundary of a stable region is composed of curves with the same constant geodesic curvature. Since any isoperimetric region is stable, the characterization of the stable regions in a surface is an interesting question in this setting. Moreover, from a physical point of view, stable regions are more realistic models since they are just local minima of perimeter, instead of global as it is the case for an isoperimetric region.

In this paper we deal with these problems in rotationally symmetric tori with decreasing Gauss curvature from the longest parallel and with a horizontal symmetry. This is a large family of surfaces, including the standard tori obtained by rotating a circle in $\mathbb{R}^{3}$ with respect to a line contained in the same plane as the circle, and at a certain distance, and certain round spheres to which hyperbolic annuli have been added. We obtain all possible stable regions that may appear, checking as well if they occur as isoperimetric.

The study of the above questions in surfaces of revolution with decreasing Gauss curvature has been treated in different works. Benjamini and Cao $\mathbf{B C}$ proved that in planes with total positive curvature less than or equal to $2 \pi$ and with Gauss curvature decreasing from a pole, the isoperimetric solutions are geodesic disks centered at the pole. Later, Morgan, Hutchings and Howards HHM2 solved the problem in the general case of decreasing Gauss curvature for planes, and also for real projective planes, annuli with an end of finite area and certain spheres, obtaining that the solutions are geodesic disks or annuli.

Date: April 6th, 2006.

2000 Mathematics Subject Classification. 49Q20, 49Q10.

Key words and phrases. Stability, isoperimetric problem.

Work partially supported by MCyT-Feder research project MTM2004-01387. 
The proofs of the results in $[\mathbf{B C},[\mathbf{P}, \mathbf{T}]$ and $\mathbf{H H M 2}$ involve different isoperimetric inequalities, which are relations between the area and the perimeter of a set. Ritoré uses in $\underline{\mathbf{R}}$ another approach, after Schmidt $\underline{\mathbf{S}}$, studying the closed embedded curves with constant geodesic curvature. Since the boundary of any isoperimetric set consists of curves of this kind, this technique allows to solve, after classifying stable regions, the isoperimetric problem in planes of revolution, spheres with an equatorial symmetry and projective planes, with decreasing and also increasing curvature, and in certain annuli with decreasing curvature.

In this paper we shall follow this second approach in order to classify the stable regions in rotationally symmetric tori with decreasing Gauss curvature from the longest parallel and with a horizontal symmetry, and we obtain in our main classification Theorem 3.3 disks bounded by constant geodesic curvature curves (symmetric with respect to such parallel), annuli bounded by two circles of revolution (symmetric or nonsymmetric with respect to the shortest parallel), unions of vertical annuli (each one bounded by two vertical geodesics), domains whose boundary is an unduloid type curve and a circle of revolution, and regions consisting of the union of a disk and a symmetric annulus. We also check that all these regions appear as stable ones in certain given surfaces.

Furthermore, we apply this classification to study the isoperimetric problem described above. One of the most interesting consequences is that an unduloid type curve may be part of the boundary of an isoperimetric region. This fact was unexpected, although this kind of sets had already appeared as solution in a work by Pedrosa and Ritoré $\underline{\mathbf{P R}}$.

We remark that along this paper, we allow the Gauss curvature $K$ to be a piecewise continuous function on $M$, which enlarges the family of surfaces considered.

Although the solutions of the isoperimetric problem in a flat torus are well-known (they are disks for small values of area, and bands, see $\mathbf{H}$ or HHM1), the same question in the standard torus of revolution has remained open for a long time, as mentioned in CHLL, an interesting work solving the double bubble problem in flat tori. From our classification of stable regions, the solution of the isoperimetric problem in such surfaces is thus reduced to numerical comparison between candidates. In Section 4 we roughly describe the isoperimetric regions in these surfaces.

We have organized this paper in several sections. In Section 1] we establish some notation and preliminaries, show the constant geodesic curvature curves and give some stability criteria for them. Section 2 is devoted to unduloid type curves: we mainly prove the existence of closed embedded stable ones in certain surfaces. In Section 3 we study the stability of regions whose boundary is composed by the curves exposed in Section 1] describing all possible stable regions in our surfaces (Theorem 3.3). Finally in Section 4, we make several comments and future directions of research regarding the isoperimetric problem.

Acknowledgments. The author would like to thank Manuel Ritoré for his continuous support and kind help during the elaboration of these notes.

\section{Preliminaries}

Let $M$ be a rotationally symmetric torus, that is, a torus endowed with a oneparameter group of intrinsic isometries. This kind of surface can be seen as the quotient of a warped product in the following sense: consider the product $\mathbb{S}^{1} \times I$, 
where $I=\left[-t_{0}, t_{0}\right]$ is a real interval, with the Riemannian metric

$$
d s^{2}=d t^{2}+f(t)^{2} d \theta^{2},
$$

where $\theta \in \mathbb{S}^{1}, t \in I$, and $f$ is a $C^{1}$ and piecewise $C^{2}$ positive function defined on $I$ (that is, $f^{\prime \prime}$ is continuous on $I$, except possibly on a finite number of points). We assume that $f\left(t_{0}\right)=f\left(-t_{0}\right)$, and $f^{\prime}\left(t_{0}\right)=f^{\prime}\left(-t_{0}\right)=0$. Then we can identify the curves $\mathbb{S}^{1} \times\left\{t_{0}\right\}$ and $\mathbb{S}^{1} \times\left\{-t_{0}\right\}$ in order to obtain the torus $M$.

We will also assume that $M$ is symmetric with respect to the curve $\mathbb{S}^{1} \times\{0\}$, that is, $f(t)=f(-t)$ for all $t \in\left[-t_{0}, t_{0}\right]$. And that the Gauss curvature $K$ is a decreasing function of the distance from $\mathbb{S}^{1} \times\{0\}$.

The horizontal curves $\mathbb{S}^{1} \times\{t\}$ have constant geodesic curvature and will be called circles of revolution or parallels. The vertical curves $\{\theta\} \times\left[-t_{0}, t_{0}\right]$ are geodesics of the metric (1.1). They will be named vertical geodesics.

In this setting, the Gauss curvature $K$ only depends on $t$, and it is given by

$$
K(t)=-\frac{f^{\prime \prime}(t)}{f(t)} .
$$

Since $f$ is only assumed to be a piecewise $C^{2}$ function, the Gauss curvature will be a piecewise continuous function in general.

Furthermore, for a circle of revolution $\mathbb{S}^{1} \times\{t\}$, the length and the geodesic curvature with respect to the normal vector $-\partial_{t}$ are given by

$$
L(t)=2 \pi f(t), \quad h(t)=\frac{f^{\prime}(t)}{f(t)} .
$$

An important remark is that the function

$$
\left(f^{\prime}\right)^{2}-f f^{\prime \prime}=(2 \pi)^{-2} L^{2}\left(K+h^{2}\right)
$$

and $K$ have the same monotone behavior.

Since $K$ is a decreasing function from $\mathbb{S}^{1} \times\{0\}$ and $M$ is not a flat torus, we have that $K(0)>0$ and $K\left(t_{0}\right)<0$. Taking into account that $\left(f^{\prime}\right)^{\prime}=-K f$, we obtain that $f^{\prime}$ is strictly negative in $\left(0, t_{0}\right)$, so that $f$ is strictly decreasing in $\left(0, t_{0}\right)$. Hence $\mathbb{S}^{1} \times\{0\}$ will be called the longest parallel, and $\mathbb{S}^{1} \times\left\{t_{0}\right\}$ will be the shortest parallel. The symmetry of $M$, with respect to $\mathbb{S}^{1} \times\{0\}$, will be referred to as horizontal symmetry.

We now show two examples of the surfaces considered in this work.

Example 1. The standard torus, obtained by rotating a circle of radius $r$, whose center is at distance a from the axis of revolution, yields one of these surfaces, with continuous Gauss curvature. In this case, the interval $I$ can be taken as $[-\pi r, \pi r]$, and the metric (1.1) is given by the function

$$
f(t)=a+r \cos (t / r),
$$

for $t \in I$, where $a>r$.

Example 2. Another example is given by the following surface: let $S$ be a sphere of radius $a$, where two identical disks, centered at each pole and at height $t^{*}$ and $-t^{*}$ from the equator, have been removed. Paste a bounded hyperbolic annulus of curvature $-b^{2}$ to each boundary component of $S$ in an appropriate way, in order to have a $C^{1}$ and piecewise $C^{2}$ surface. Finally, by identifying the two remaining boundary components of the annuli we obtain one of our surfaces, now with piecewise continuous Gauss 
curvature. Here the interval $I$ is $[-d / b, d / b]$ and the metric (1.1) is provided by the function

$$
f(t)=\frac{1}{a} \cos (a t), t \in\left[0, t^{*}\right],
$$

in the upper spherical piece, and

$$
f(t)=c \cosh (d-b t), t \in\left[t^{*}, d / b\right],
$$

in the upper hyperbolic piece. By the $C^{1}$-differentiability of $f$ in $t^{*}$, it turns that $b$ must be greater than a $\tan \left(a t^{*}\right)$ and then

$$
\begin{aligned}
c^{2} & =\frac{1}{a^{2}} \cos ^{2}\left(a t^{*}\right)-\frac{1}{b^{2}} \sin ^{2}\left(a t^{*}\right), \\
d & =b t^{*}+\cosh ^{-1}\left(\frac{\cos \left(a t^{*}\right)}{a c}\right) .
\end{aligned}
$$

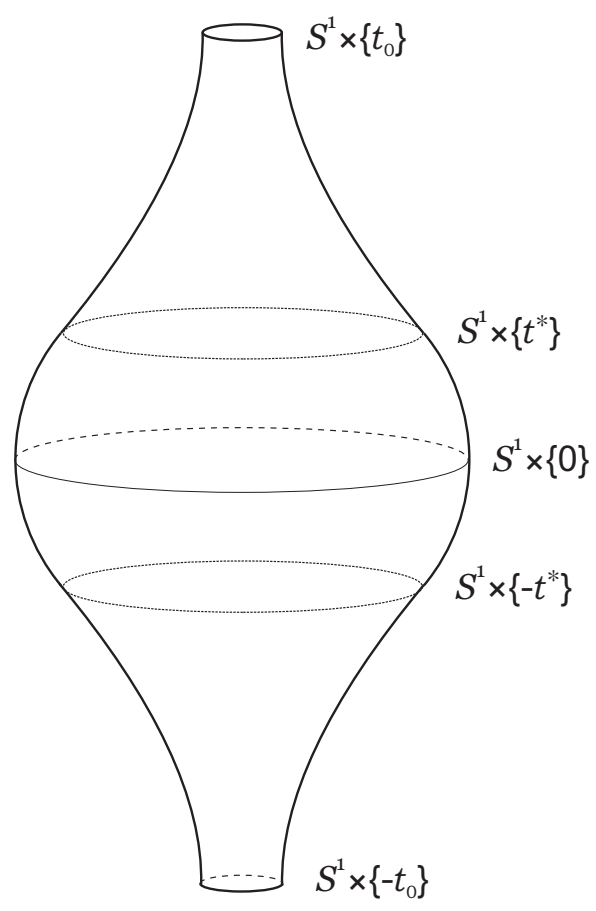

Figure 1. One of the surfaces described in Example 2 built from a sphere and two hyperbolic annuli

Remark 1.1. This second example can also be considered with the function

$$
f(t)=\cos (a t), t \in\left[0, t^{*}\right]
$$

in the upper spherical piece, obtaining other kind of surfaces. These functions are associated to singular orbifolds with constant Gauss curvature.

1.1. Constant geodesic curvature curves. In this subsection we will describe the closed embedded curves with constant geodesic curvature appearing in our surfaces. In the warped product $\mathbb{S}^{1} \times I$, these curves have been well studied in $\underline{\mathbf{R}}$ when $f$ is $C^{2}$, by using a result by Osserman [O Lemma 7]. However, such a result is also valid when $f$ is piecewise $C^{2}$, and so we can extend most of the consequences to our case. 
Let $\gamma(s)=(\theta(s), t(s))$ be a curve parametrized by arc-length $s$ in $S^{1} \times I$. Denote by $d \gamma / d s$ the tangent vector to $\gamma$, and by $\sigma$ the oriented angle $\angle\left(\partial_{t}, d \gamma / d s\right)$. Consider the unit normal vector to $\gamma$ given by

$$
N=\frac{\cos \sigma}{f(t)} \partial_{\theta}-\sin \sigma \partial_{t},
$$

and let $h$ be the geodesic curvature of $\gamma$ with respect to $N$.

Proposition 1.2. ( $\mathbf{R}$ Prop. 1.1]) With the notation above, the curve $\gamma$ satisfies the following system of ordinary differential equations

$$
\begin{aligned}
& \frac{d t}{d s}=\cos \sigma, \\
& \frac{d \theta}{d s}=\frac{\sin \sigma}{f(t)}, \\
& \frac{d \sigma}{d s}=h-\frac{f^{\prime}(t)}{f(t)} \sin \sigma .
\end{aligned}
$$

Moreover, if $h$ is constant then, for any $c \in I$, the function

$$
f(t) \sin \sigma-h \int_{c}^{t} f(\xi) d \xi
$$

is constant over any solution of (1.2).

Remark 1.3. In view of the system (1.2), it can be checked that a constant geodesic curvature curve $\gamma$ in $\mathbb{S}^{1} \times I$ is periodic with respect to any of the critical points of $t_{\mid \gamma}$.

The function (1.3) is called a first integral of (1.2), and allows, as in $\mathbf{R}$, to classify the closed embedded curves with constant geodesic curvature in $M$, obtaining the following

Theorem 1.4. Let $M$ be a rotationally symmetric torus with decreasing Gauss curvature from the longest parallel. Assume also that $M$ has a horizontal symmetry. Let $C$ be a connected closed embedded curve in $M$ with constant geodesic curvature.

Then $C$ is a circle of revolution, a vertical geodesic, a nodoid type curve or an unduloid type curve.

Consider a constant geodesic curvature curve $C$ in $\mathbb{S}^{1} \times I$, with a strict maximum $t\left(s_{0}\right)$ of the $t$-coordinate, and let $s_{1}>s_{0}$ be the next critical point. If $\sin \left(\sigma\left(s_{1}\right)\right)=1$, the curve will be called unduloid type curve, and it can be checked that is a periodic graph over $\theta$. Otherwise, if $\sin \left(\sigma\left(s_{1}\right)\right)=-1$, the curve will be a nodoid type curve and will present points with vertical tangent vector.

We shall summarize some properties of circles of revolution in a warped product $\mathbb{S}^{1} \times I$.

Lemma 1.5. The geodesic curvature of circles of revolution $h(t)$, computed with respect to the normal vector $-\partial_{t}$, satisfies the following properties:

i) $h(t)$ is an antisymmetric function on $\left[-t_{0}, t_{0}\right]$.

ii) $h(t)$ is increasing in the interval where $\left(f^{\prime}\right)^{2}-f f^{\prime \prime} \leqslant 0$, and decreasing where $\left(f^{\prime}\right)^{2}-f f^{\prime \prime} \geqslant 0$.

Remark 1.6. Note that $h(0)=0$, and $h\left(-t_{0}\right)=h\left(t_{0}\right)=0$, since $f^{\prime}\left(t_{0}\right)=0$. Moreover, $\left[\left(f^{\prime}\right)^{2}-f f^{\prime \prime}\right]\left(-t_{0}\right)=f\left(-t_{0}\right)^{2} K\left(-t_{0}\right)<0$, and $\left[\left(f^{\prime}\right)^{2}-f f^{\prime \prime}\right](0)=f(0)^{2} K(0)>0$. Then, as $K(t)$ is increasing in $\left(-t_{0}, 0\right)$, Lemma 1.5 gives that $h(t)$ is increasing from $-t_{0}$ until the point in $\left(-t_{0}, 0\right)$ where $\left(f^{\prime}\right)^{2}-f f^{\prime \prime}$ vanishes, and decreasing from that point until 0 . Consequently, $h(t)$ is positive in $\left[-t_{0}, 0\right]$, and negative in $\left[0, t_{0}\right]$. 


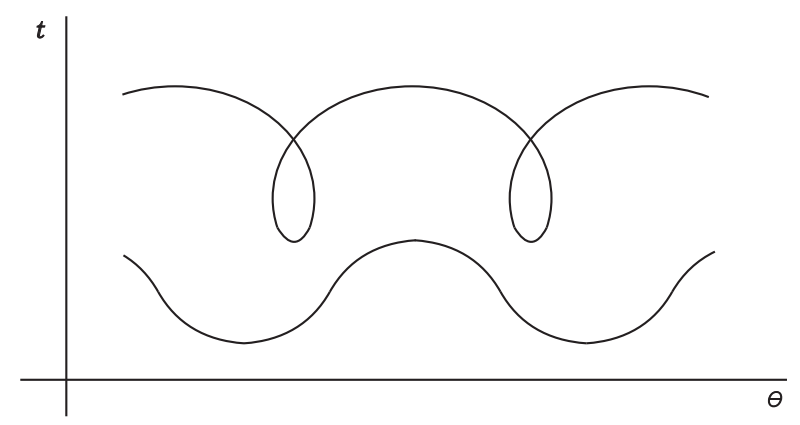

Figure 2. Nodoid and unduloid type curves in $\mathbb{S}^{1} \times I$

We will now treat when nodoid and unduloid type curves yield closed embedded curves. We first define the period of an unduloid type curve as the $\theta$-distance between two consecutive maxima (or minima) points of the $t$-coordinate.

Lemma 1.7. ([ $\mathbf{R}$, Prop. 1.3]) Let $C$ be a curve with constant geodesic curvature in a warped product $\mathbb{S}^{1} \times I$.

i) If $C$ is a nodoid type curve, it yields a closed embedded curve if and only if the maximum and the minimum of $t_{C}$ are in the same vertical line.

ii) If $C$ is an unduloid type curve, it yields a closed embedded curve if and only if the period of $C$ is equal to $2 \pi / k$, with $k \in \mathbb{N}$.

1.2. Stability and the index form. Consider a curve $C$ with constant geodesic curvature $h$, not necessarily connected, enclosing a certain area of $M$. Then, it is well-known that $C$ is a critical point for the length functional, for area-preserving variations [BP]. We shall say that $C$ is stable if it is a local minimum of perimeter for any variation of $C$ with fixed area enclosed. If $C$ is contained in an open region where $K$ is continuous, then the second derivative of length is given by

$$
I(u)=-\int_{C} u\left\{\frac{d^{2} u}{d s^{2}}+\left(K+h^{2}\right) u\right\} d s,
$$

where $u: C \rightarrow \mathbb{R}$ is the normal component of the vector field associated to the variation. In this case, we have that the stability of $C$ equivalent to

$$
I(u) \geqslant 0, \text { for any function } u \text { such that } \int_{C} u d s=0 .
$$

A set $\Omega \subset M$ is called a stable region if $\partial \Omega$ is an embedded stable curve with constant geodesic curvature with respect to the inner normal. This means that the boundary of a stable region is a second order local minimum for the perimeter when keeping constant the area enclosed. It is clear that any isoperimetric region is stable.

Hereafter, the quadratic form of (1.4) will be called the index form, and the associated self-adjoint operator

$$
J(u)=\frac{d^{2} u}{d s^{2}}+\left(K+h^{2}\right) u
$$

will be called the Jacobi operator.

A function $u: C \rightarrow \mathbb{R}$ satisfying $J(u)=0$ is a Jacobi function. For instance, the normal component $u=\left\langle N, \partial_{\theta}\right\rangle=f(t) \cos \sigma$ of the Killing vector field $\partial_{\theta}$ is always a Jacobi function. Moreover, Jacobi functions also arise from variations of $C$ keeping 
constant the geodesic curvature along the deformation, since for a variation with normal component $u$ we have (see BGS

$$
\left.\frac{d h}{d t}\right|_{t=0}=u^{\prime \prime}+\left(K+h^{2}\right) u=J(u) .
$$

Given a Jacobi function $u$, a nodal region is a connected component of the complementary in $C$ of the set $\{x \in C: u(x)=0\}$. By Courant's Nodal Domain Theorem it follows that stable connected curves have at most two nodal regions (see $\mathbf{C h}$ Ch. I, pag. 19]).

The next lemma gives a stability condition for circles of revolution.

Lemma 1.8. ( $\underline{\mathbf{R}}$ Lemma 1.6]) A circle of revolution $\mathbb{S}^{1} \times\{t\}$ is stable if and only if

$$
\left(K+h^{2}\right)(t) \leqslant \frac{4 \pi^{2}}{L^{2}(t)}, \quad \text { or equivalently } \quad\left[\left(f^{\prime}\right)^{2}-f f^{\prime \prime}\right](t) \leqslant 1 .
$$

Remark 1.9. Consider $\tilde{t} \in I$ such that $\left[\left(f^{\prime}\right)^{2}-f f^{\prime \prime}\right](\tilde{t})=1$. Then the Jacobi operator for the parallel $\mathbb{S}^{1} \times\{\tilde{t}\}$ is

$$
J(u)=u^{\prime \prime}+\frac{1}{f(\tilde{t})^{2}} u .
$$

It is easy to check that $\sin (\theta(s))$ and $\cos (\theta(s))$ are Jacobi functions of $\mathbb{S}^{1} \times\{\tilde{t}\}$. Moreover, this parallel is the unique stable one with two independent Jacobi functions.

The following lemma treats stable nodoid type curves in $M$. When they are closed embedded curves, they bound disks in the surface.

Lemma 1.10. Let $C$ be a closed embedded stable nodoid type curve in $M$, not contained in a region with constant Gauss curvature. Then $C$ intersects symmetrically $\mathbb{S}^{1} \times\{0\}$.

Proof. We give an sketch of the proof, see $\underline{\mathbf{R}}$ Lemmata 2.3 and 3.4] for details. As $C$ is closed and embedded, an analytical reasoning [O Lemma 7] will give that it cannot be contained in a region of $M$ with (non-constant) monotone Gauss curvature. Therefore $C$ will intersect $\mathbb{S}^{1} \times\{0\}$, or $\mathbb{S}^{1} \times\left\{t_{0}\right\}$. By reflecting the curve, another application of the same reasoning will show that $C$ is necessarily symmetric with respect to $\mathbb{S}^{1} \times\{0\}$. Finally, if $C$ meets $\mathbb{S}^{1} \times\left\{t_{0}\right\}$, a suitable function in the index form yield instability.

\section{Stable unduloid type CURVES}

In this section we will consider unduloid type curves. Our aim is to prove that closed embedded stable ones may occur in certain rotationally symmetric tori. We remark that these curves did not appear in any of the surfaces studied in $[\mathbf{R}$. Stability will be obtained by using a result from $\mathbf{H L}$, which involves variations of unduloid type curves by constant geodesic curvature, and requires the study of the eigenvalue problem associated to the Jacobi operator.

First, existence of closed embedded unduloid type curves in some surfaces is guaranteed from the following result.

Lemma 2.1. Let $M$ be a rotationally symmetric torus with a horizontal symmetry and decreasing Gauss curvature $K$ from the longest parallel. Assume also that there exists a parallel $\mathbb{S}^{1} \times\{\tilde{t}\}$ in $M$ satisfying $\left[\left(f^{\prime}\right)^{2}-f f^{\prime \prime}\right](\tilde{t})=1$, and such that $K$ is smooth and strictly decreasing in a neighborhood of $\mathbb{S}^{1} \times\{\tilde{t}\}$. 
Then there are closed embedded unduloid type curves in $M$, close to $\mathbb{S}^{1} \times\{\tilde{t}\}$.

Proof. Recall that any unduloid type curve is a graph over $\theta$. Then, writing $t=$ $t(\theta), \sigma=\sigma(\theta)$, the system of ordinary differential equations obtained from (1.2) which satisfies such a curve is

$$
\begin{aligned}
& \frac{d t}{d \theta}=f(t) \cot \sigma \\
& \frac{d \sigma}{d \theta}=h \frac{f(t)}{\sin \sigma}-f^{\prime}(t) .
\end{aligned}
$$

Let $\gamma(\theta, T, h)=(\theta, t(\theta, T, h), \sigma(\theta, T, h))$ denote the solution of (2.1), with initial conditions $t(0, T, h)=T, \sigma(0, T, h)=\pi / 2$ and geodesic curvature $h$. For $T>\tilde{t}$, this means that $T$ is the maximum value achieved by $t(\theta, T, h)$. Call $\tilde{h}=f^{\prime}(\tilde{t}) / f(\tilde{t})$.

Observe that for $T=\tilde{t}, h=\tilde{h}$, the solution of 2.1) is $(\theta, \tilde{t}, \pi / 2)$, the circle of revolution $\mathbb{S}^{1} \times\{\tilde{t}\}$. We are going to see that for $T$ close enough to $\tilde{t}$, there exist unduloid type curves with period $2 \pi$, so by Lemma 1.7 they will be closed and embedded.

Note that for $(T, h)$ close enough to $(\tilde{t}, \tilde{h})$, the curves $\gamma(\theta, T, h)$ will be unduloid type ones or circles of revolution by the first integral (1.3), since $\sin \sigma>0$.

Let us define the function $F$ by

$$
F(T, h)=\sigma(\pi, T, h) .
$$

Note that if $F(T, h)=\pi / 2$, the corresponding unduloid type curve $\gamma(\theta, T, h)$ has period $2 \pi$. Clearly $F(\tilde{t}, \tilde{h})=\pi / 2$. We want to find functions $h(T)$ such that for $T$ close enough to $\tilde{t}, F(T, h(T))=\pi / 2$. Let us compute the partial derivatives of $F$ at $(\tilde{t}, \tilde{h})$, which coincide with the partial derivatives of $\sigma$ at $(\pi, \tilde{t}, \tilde{h})$.

We shall denote by $t_{T}(\theta), t_{h}(\theta)$, (resp. $\left.\sigma_{T}(\theta), \sigma_{h}(\theta)\right), \ldots$, the partial derivatives of the function $t(\theta, T, h)$ (resp. $\sigma(\theta, T, h))$ at $(\theta, \tilde{t}, \tilde{h})$. Then, Taylor developments at $(\theta, \tilde{t}, \tilde{h})$ give

$$
\begin{gathered}
t(\theta, T, h)=\tilde{t}+(T-\tilde{t}) t_{T}(\theta)+(h-\tilde{h}) t_{h}(\theta)+(T-\tilde{t})(h-\tilde{h}) t_{T h}(\theta)+ \\
\frac{1}{2}(T-\tilde{t})^{2} t_{T T}(\theta)+\frac{1}{2}(h-\tilde{h})^{2} t_{h h}(\theta)+\frac{1}{6}(T-\tilde{t})^{3} t_{T T T}(\theta)+\ldots, \\
\sigma(\theta, T, h)=\pi / 2+(T-\tilde{t}) \sigma_{T}(\theta)+(h-\tilde{h}) \sigma_{h}(\theta)+(T-\tilde{t})(h-\tilde{h}) \sigma_{T h}(\theta)+ \\
\frac{1}{2}(T-\tilde{t})^{2} \sigma_{T T}(\theta)+\frac{1}{2}(h-\tilde{h})^{2} \sigma_{h h}(\theta)+\frac{1}{6}(T-\tilde{t})^{3} \sigma_{T T T}(\theta)+\ldots
\end{gathered}
$$

Moreover, from the definitions of $t(\theta, T, h)$ and $\sigma(\theta, T, h)$, we have that $t_{T}(0)=1$, $t_{h}(0)=t_{T h}(0)=t_{T T}(0)=t_{h h}(0)=t_{T T T}(0)=0, \sigma_{T}(0)=\sigma_{h}(0)=\sigma_{T h}(0)=$ $\sigma_{T T}(0)=\sigma_{h h}(0)=\sigma_{T T T}(0)=0$.

From (2.1), considering the second equation as

$$
\frac{d \sigma}{d \theta}=(h-\tilde{h}) \frac{f(t)}{\sin \sigma}+\tilde{h} \frac{f(t)}{\sin \sigma}-f^{\prime}(t),
$$

and Taylor developments of the involved functions, we get

$$
\begin{aligned}
\frac{d t_{T}}{d \theta} & =-f(\tilde{t}) \sigma_{T}, \\
\frac{d \sigma_{T}}{d \theta} & =\frac{t_{T}}{f(\tilde{t})},
\end{aligned}
$$

which gives $t_{T}(\theta)=\cos (\theta), \sigma_{T}(\theta)=\sin (\theta) / f(\tilde{t})$. 
In the same way,

$$
\begin{aligned}
\frac{d t_{h}}{d \theta} & =-f(\tilde{t}) \sigma_{h}, \\
\frac{d \sigma_{h}}{d \theta} & =f(\tilde{t})+\frac{t_{h}}{f(\tilde{t})},
\end{aligned}
$$

obtaining $t_{h}(\theta)=f(\tilde{t})^{2}(\cos (\theta)-1), \sigma_{h}(\theta)=f(\tilde{t}) \sin (\theta)$.

Therefore, the gradient of $F$ at $(\tilde{t}, \tilde{h})$ is equal to

$$
\nabla F(\tilde{t}, \tilde{h})=\left(\sigma_{T}(\pi), \sigma_{h}(\pi)\right)=(0,0) .
$$

We now compute the hessian of $F$ at $(\tilde{t}, \tilde{h})$. From the equations above we obtain the following systems.

$$
\begin{gathered}
\frac{d t_{T T}}{d \theta}=-f(\tilde{t})\left(\sigma_{T T}-2 \tilde{h} t_{T} \sigma_{T}\right) \\
\frac{d \sigma_{T T}}{d \theta}=f(\tilde{t})\left(\frac{1}{f(\tilde{t})^{2}} t_{T T}+K^{\prime}(\tilde{t}) t_{T}^{2}+\tilde{h} \sigma_{T}^{2}\right) \\
\frac{d t_{h h}}{d \theta}=-f(\tilde{t})\left(\sigma_{h h}+2 \tilde{h} t_{h} \sigma_{h}\right) \\
\frac{d \sigma_{h h}}{d \theta}=f(\tilde{t})\left(2 \tilde{h} t_{h}+\frac{1}{f(\tilde{t})^{2}} t_{h h}+K^{\prime}(\tilde{t}) t_{h}^{2}+\tilde{h} \sigma_{h}^{2}\right) \\
\frac{d t_{T h}}{d \theta}=-f(\tilde{t})\left(\sigma_{T h}+\tilde{h}\left(t_{T} \sigma_{h}+t_{h} \sigma_{T}\right)\right) \\
\frac{d \sigma_{T h}}{d \theta}=f(\tilde{t})\left(\tilde{h} t_{T}+\frac{1}{f(\tilde{t})^{2}} t_{T h}+K^{\prime}(\tilde{t}) t_{h} t_{T}+\tilde{h} \sigma_{T} \sigma_{h}\right) .
\end{gathered}
$$

Solving these systems, we get that $\sigma_{T T}(\pi)=0, \sigma_{T h}(\pi)=\rho / 2$ and $\sigma_{h h}(\pi)=\rho f(\tilde{t})^{2}$, where $\rho=\pi f(\tilde{t})^{3} K^{\prime}(\tilde{t})$, and so the hessian of $F$ at $(\tilde{t}, \tilde{h})$ is given by

$$
\nabla^{2} F(\tilde{t}, \tilde{h})=\left(\begin{array}{cc}
0 & \rho / 2 \\
\rho / 2 & \rho f(\tilde{t})^{2}
\end{array}\right) .
$$

Therefore $\nabla^{2} F(\tilde{t}, \tilde{h})$ is non-degenerate, since $K$ is a strictly decreasing function.

Then $(\tilde{t}, \tilde{h})$ is a non-degenerate critical point of $F$. By applying Morse's Lemma Mi Lemma 2.2] we obtain two planar curves $\alpha_{c}, \alpha_{o}$ with $\alpha_{c}(0)=\alpha_{o}(0)=(\tilde{t}, \tilde{h})$ and such that, for any point $(T, h)$ close to $(\tilde{t}, \tilde{h})$ satisfying $F(T, h)=\pi / 2$, it follows that $(T, h)$ lies on the trace of $\alpha_{c} \cup \alpha_{o}$. Let us study the tangent vectors of both curves at the origin. Denoting by $\alpha$ one of those curves, we know that $F \circ \alpha=\pi / 2$. Differentiating such an equality we obtain $\left\langle\nabla F_{\alpha}, \alpha^{\prime}\right\rangle=0$. Differentiating once again and evaluating at the origin, we have

$$
\alpha^{\prime}\left\langle\nabla F_{(\tilde{t}, \tilde{h})}, \alpha^{\prime}\right\rangle=\nabla^{2} F_{(\tilde{t}, \tilde{h})}\left(\alpha^{\prime}, \alpha^{\prime}\right)=0
$$

From (2.6), we get $\alpha_{c}^{\prime}(0)=\left(1,-1 / f(\tilde{t})^{2}\right)$ and $\alpha_{o}^{\prime}(0)=(1,0)$. Hence both curves can be written in terms of $T$ near $\tilde{t}$, because the tangent vectors are not vertical. So there exist two functions $h_{c}(T), h_{o}(T)$ defined in a neighborhood of $\tilde{t}$ such that $\left\{\left(T, h_{c}(T)\right)\right\}$ is the trace of the curve $\alpha_{c}$, and $\left\{\left(T, h_{o}(T)\right)\right\}$ is the trace of $\alpha_{o}$. Initial conditions $\left(T, h_{c}(T)\right)$ in (2.1) yield circles of revolution, and $\left(T, h_{o}(T)\right)$ give unduloid type curves as solutions (note that $h_{c}^{\prime}(\tilde{t})=-1 / f(\tilde{t})^{2}$ and $\left.h_{o}^{\prime}(\tilde{t})=0\right)$. 
Since

$$
F\left(T, h_{o}(T)\right)=\left(F \circ \alpha_{o}\right)(s)=\pi / 2,
$$

we conclude that the unduloid type curves $\gamma\left(\theta, T, h_{o}(T)\right)$ have period $2 \pi$, and so they are closed and embedded.

We now focus on the stability of closed embedded unduloid type curves. A first remark is that stable ones will present a unique maximum point (and therefore a unique minimum point) for the $t$-coordinate. Otherwise, the normal component of the rotations vector field will have more than two nodal regions, yielding instability by applying Courant's Nodal Domain Theorem. In view of Lemma 1.7 this fact is equivalent to that the period of closed embedded stable unduloid type curves equals $2 \pi$.

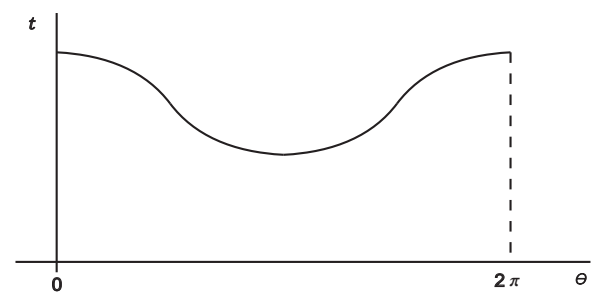

Figure 3. A closed embedded stable unduloid type curve in $\mathbb{S}^{1} \times I$

The following lemma states another necessary condition for stability.

Lemma 2.2. Let $C$ be a closed embedded stable unduloid type curve in $M$, not contained in the region $\left\{\left(f^{\prime}\right)^{2}-f f^{\prime \prime}=1\right\}$. Then there are points of $C$ satisfying $\left(f^{\prime}\right)^{2}-f f^{\prime \prime}>1$, and other ones satifying $\left(f^{\prime}\right)^{2}-f f^{\prime \prime}<1$.

Proof. Suppose first that $C$ lies in a region where $\left(f^{\prime}\right)^{2}-f f^{\prime \prime} \leqslant 1$. Then, by $\mathbb{R}$ Lemma 2.3], its period is greater than $2 \pi$, and so it is not a closed embedded curve by Lemma 1.7 Assume now that $C$ lies in a region where $\left(f^{\prime}\right)^{2}-f f^{\prime \prime} \geqslant 1$. Then its period is less than $2 \pi$ (see $\mathbf{R}$ Lemma 2.13] for details), and so $C$ will present more than one maximum point for the $t$-coordinate, contradicting stability.

In view of the Jacobi operator (1.5), we can consider the eigenvalue problem associated to a closed embedded unduloid type curve $C$ in $M$ :

$$
J(u)+\lambda u=0,
$$

with $u: C \rightarrow \mathbb{R}$ a $C^{2}$ function.

We will call eigenvalues to the real numbers $\lambda$ for which there exist functions satisfying (2.8). Such functions will be named eigenfunctions associated to $\lambda$. We recall some well-known general facts about this problem.

Lemma 2.3. ([CL Chapter 8, Theorem 2.1], Ch ) Given a curve $C$, the eigenvalues associated to the Jacobi operator form an increasing sequence $\left\{\lambda_{i}\right\}_{i \geqslant 1}$. Furthermore, the space of eigenfunctions $V_{\lambda_{i}}$ associated to $\lambda_{i}$, when considering an eigenvalue problem with boundary conditions, is one-dimensional and each $\phi_{i} \in V_{\lambda_{i}}$ has exactly $i-1$ zeros.

We will now show some results regarding the eigenvalue problem (2.8), necessary for our purposes. 
Lemma 2.4. Let $C$ be a closed embedded unduloid type curve in $M$. Then the first eigenvalue for the Jacobi operator associated to $C$ is negative, and the second eigenvalue is non-positive.

Proof. Consider the normal component $u$ of the rotations vector field on $M$, which is a Jacobi function. Therefore the restriction of $u$ to $C$ is an eigenfunction for the associated eigenvalue problem (2.8) with zero eigenvalue.

It is easy to check that $u$ gives at least two nodal regions in $C$, since the period of $C$ must be $2 \pi / k$, for some $k \in \mathbb{N}$, by Lemma 1.7. Therefore, by applying Courant's Nodal Domain Theorem, the statement follows.

Remark 2.5. Closed embedded stable curves have non-negative second eigenvalue $\lambda_{2}$ for the above eigenvalue problem (2.8). From Lemma [2.4] it follows that closed embedded stable unduloid type curves in $M$ verify $\lambda_{2}=0$.

Remark 2.6. Given $C$ a nodoid type curve in $M$, the same reasoning as above shows that Lemma 2.4 also holds when $C$ is closed and embedded.

Let $\lambda$ be an eigenvalue of (2.8) for a given closed embedded unduloid type curve $C$, with associated eigenfunction $u$. Call fundamental piece of $C$ to any region of $C$ delimited by a maximum point of the $t$-coordinate, and the consecutive minimum point. By considering the vertical reflection with respect to a maximum point of the $t$-coordinate of $C$, we can express $u=u_{s}+u_{a}$, where $u_{s}$ is a symmetric eigenfunction satisfying the Neumann boundary condition in any fundamental piece $C^{\prime}$ of $C$, and $u_{a}$ is an antisymmetric eigenfunction satisfying the Dirichlet boundary condition in $C^{\prime}$, both of them with eigenvalue $\lambda$. We will also name $\lambda_{i}^{N}\left(C^{\prime}\right), \lambda_{i}^{D}\left(C^{\prime}\right)$ to the eigenvalues associated to $C^{\prime}$ for the Neumann and Dirichlet eigenvalue problem, respectively.

Recall that $\lambda_{1}<0$ by Lemma 2.4 Therefore, the above reasoning leads to a negative Dirichlet eigenvalue in $C^{\prime}$, which is not possible since $\lambda_{1}^{D}\left(C^{\prime}\right)=0$. Then $u_{a}=0$, and so $u=u_{s}$. Consequently, $\lambda_{1}$ coincides with $\lambda_{1}^{N}\left(C^{\prime}\right)$.

Now consider the second eigenvalue $\lambda_{2}$ in $C$. If it is negative, then the curve is unstable, and moreover, an analogous treatment will give $\lambda_{2}=\lambda_{2}^{N}\left(C^{\prime}\right) \underline{\mathbf{B B}}$. On the other hand, if $\lambda_{2} \geqslant 0$, then necessarily $\lambda_{2}=0$ by Lemma 2.4

We will now study the period of unduloid type curves, not necessarily closed and embedded. For $\gamma\left(\theta, T_{0}, h\right)$, such a period is defined as the $\theta$-distance between two consecutive maximum points (or minimum points) for the $t$-coordinate. If we move slightly the maximum point $T$, keeping constant the geodesic curvature, the period of $\gamma(\theta, T, h)$ only depends on $T$, and then the derivative of the period with respect to $T$, at $T=T_{0}$, can be considered.

Lemma 2.7. ([PR Corollary 2.8]) Consider an unduloid type curve $C$ in $M$, close enough to $\mathbb{S}^{1} \times\{\tilde{t}\}$. Let $C^{\prime}$ be a fundamental piece of $C$. Then the second eigenvalue $\lambda_{2}^{N}\left(C^{\prime}\right)$ of the Neumann problem for the Jacobi operator in $C^{\prime}$ is positive if and only if the derivative of the period with respect to the maximum point is positive.

Proof. Call $C=\gamma\left(\theta, T_{0}, h\right)$ and consider the variation by unduloid type curves given by $\gamma(\theta, T, h)$, with $T$ close to $T_{0}$ and $h$ fixed. It can be checked that the associated variational function $u$ is a Jacobi function satisfying $u(0)=1, u^{\prime}(0)=0$ and $u^{\prime \prime}(0)<0$, and so $u^{\prime}(\varepsilon)<0$ for positive $\varepsilon$ close to 0 .

Assume first that the derivative is positive, and let us find a convenient expression of it. Let $\theta_{2}>0$ be the first instant where the $t$-coordinate of $C$ achieves a minimum. 
Therefore $\sigma^{\prime}\left(\theta_{2}\right)<0$, and $\left[0, \theta_{2}\right]$ yields a fundamental piece of $C$, say $C^{\prime}$. By applying Implicit Function Theorem to $\sigma(\theta, T)$ at $\left(\theta_{2}, T_{0}\right)$, we obtain a function $\theta(T)$ such that $\sigma(\theta(T), T)=\pi / 2$, and so $\theta(T)$ gives (half) the period of $\gamma(\theta, T, h)$. Straightforward calculations for computing $\theta^{\prime}(T)$ show that the desired derivative equals

$$
\frac{2 u^{\prime}\left(\theta_{2}\right)}{f\left(t\left(\theta_{2}, T_{0}\right)\right) \sigma^{\prime}\left(\theta_{2}\right)} .
$$

Since we are assuming that such a value is positive, then we have $u^{\prime}\left(\theta_{2}\right)<0$.

We will focus now on the interval $\left(0, \theta_{2}\right)$. Observe that $u$ will have at most one zero on such interval. Otherwise, by considering two consecutive zeroes $\theta_{3}, \theta_{4}$, it turns that the first eigenvalue of the Dirichlet problem in $\left(\theta_{3}, \theta_{4}\right)$ is zero (recall that $u$ is a Jacobi function), and then $\lambda_{1}^{D}\left(C^{\prime}\right)$ will be strictly negative, by the monotone property of eigenvalues $\mathbf{C h}$, which is contradictory.

On the other hand, $u^{\prime}$ does not vanish in $\left(0, \theta_{2}\right)$. Since $u^{\prime}(\varepsilon)<0$ for $\varepsilon>0$, and $u^{\prime}\left(\theta_{2}\right)<0$, if $u^{\prime}$ has a zero then $u^{\prime \prime}$ will have two zeroes. As $u^{\prime \prime}+\left(K+h^{2}\right) u=0$, it follows that $u$ will vanish twice in $\left(0, \theta_{2}\right)$, and we would proceed as above.

Let $\theta_{3}$ be the first zero of $u^{\prime}$ greater than $\theta_{2}$. As $u^{\prime}$ is strictly negative in $\left(0, \theta_{3}\right)$ and vanishes at the extremes, then $u^{\prime \prime}$ changes its sign in $\left(0, \theta_{3}\right)$. It follows that $u$ is strictly decreasing and vanishes only once in $\left(0, \theta_{3}\right)$. By Lemma 2.3 $u$ is the second eigenfunction of the Neumann problem in $\left.C\right|_{\left(0, \theta_{3}\right)}$, and the associated second Neumann eigenvalue is zero. By the monotone property of eigenvalues we conclude that $\lambda_{2}^{N}\left(C^{\prime}\right)>0$.

Assume now that $\lambda_{2}^{N}\left(C^{\prime}\right)>0$. If the derivative of the period is negative, by applying directly $\left[\mathbf{P R}\right.$ Cor. 2.8] we obtain that $\lambda_{2}^{N}\left(C^{\prime}\right)<0$, a contradiction. If the derivative of the period is zero, same reasoning as above leads to $\lambda_{2}^{N}\left(C^{\prime}\right)=0$, again a contradiction. So the derivative must be positive and the statement follows.

The next lemma determines the sign of the derivative of the period of closed embedded unduloid type curves (close enough to $\mathbb{S}^{1} \times\{\tilde{t}\}$ ) by means of the following intrinsic condition on the surface.

Lemma 2.8. Consider a surface $\mathbb{S}^{1} \times I$ under the conditions of Lemma[2.1],

Then, the derivative of the period of closed embedded unduloid type curves with respect to $T$ is strictly positive, for $T>\tilde{t}$ close enough, if and only if

$$
3 K(\tilde{t})(1-f(\tilde{t}))+3 f(\tilde{t})^{2}\left(\tilde{h} K^{\prime}(\tilde{t})-K^{\prime \prime}(\tilde{t})\right)+5 f(\tilde{t})^{4} K^{\prime}(\tilde{t})^{2}>0 .
$$

Proof. Fix $T_{0}>\tilde{t}$ close enough, and $h_{o}\left(T_{0}\right)$ as geodesic curvature in order to compute the desired derivative at $T=T_{0}$. It is clear that such a derivative will be strictly positive if and only if the derivative of the period at $T=\tilde{t}$ is strictly positive, for constant geodesic curvature $\tilde{h}=h(\tilde{t})$ (note that the derivative will preserve the same sign in an approppiate neighborhood of $(\tilde{t}, \tilde{h}))$.

Denote by $t(\theta, T), \sigma(\theta, T)$ to the solutions of (2.1) with initial conditions $t(0, T)=$ $T, \sigma(0, T)=\pi / 2$, and geodesic curvature $\tilde{h}$.

We search a function $\theta: \mathbb{R} \rightarrow \mathbb{R}$ verifying $\sigma(\theta(T), T)=\pi / 2$. If such a function exists, $\theta(T)$ will give the (half) period of $\gamma(\theta, T, \tilde{h})$.

Consider the auxiliar function $F$ given by

$$
\sigma(\theta, T)-\pi / 2=(T-\tilde{t}) F(\theta, T),
$$


and extended continuously in $(\theta, \tilde{t})$. That is,

$$
F(\theta, \tilde{t})=\sigma_{T}(\theta, \tilde{t})=\frac{\sin (\theta)}{f(\tilde{t})} .
$$

Observe that $F(\pi, \tilde{t})=0$. Moreover, for $T \neq \tilde{t}$, we have that $F(\theta, T)=0$ if and only if $\sigma(\theta, T)=\pi / 2$.

From Taylor development of $\sigma(\theta, T)$ of Lemma 2.1 we have

$$
F(\theta, T)=\sigma_{T}(\theta)+\frac{1}{2} \sigma_{T T}(\theta)(T-\tilde{t})+\frac{1}{6} \sigma_{T T T}(\theta)(T-\tilde{t})^{2}+O\left((T-\tilde{t})^{3}\right) .
$$

By differentiating (2.11),

$$
\frac{\partial F}{\partial \theta}(\pi, \tilde{t})=\sigma_{T}^{\prime}(\pi)=\frac{-1}{f(\tilde{t})} \neq 0 .
$$

By applying the Implicit Function Theorem, there exists $\theta(T)$, for $T$ close to $\tilde{t}$, such that $\theta(\tilde{t})=\pi$, and

$$
F(\theta(T), T)=0,
$$

equivalently $\sigma(\theta(T), T)=\pi / 2$. So this function $\theta(T)$ gives the period of the unduloid type curve $\gamma(\theta, T, \tilde{h})$.

We now compute the derivative of $\theta(T)$. Since

$$
\theta(T)=\theta(\tilde{t})+\theta^{\prime}(\tilde{t})(T-\tilde{t})+\frac{1}{2} \theta^{\prime \prime}(\tilde{t})(T-\tilde{t})^{2}+O\left((T-\tilde{t})^{3}\right),
$$

we have

$$
\theta^{\prime}(T)=\theta^{\prime}(\tilde{t})+\theta^{\prime \prime}(\tilde{t})(T-\tilde{t})+O\left((T-\tilde{t})^{2}\right) .
$$

From (2.12) it follows that

$$
\theta^{\prime}(T)=-\frac{\partial F / \partial T}{\partial F / \partial \theta}(\theta(T), T) .
$$

Evaluating in $T=\tilde{t}$, and taking into account (2.11), we have

$$
\theta^{\prime}(\tilde{t})=\frac{-\sigma_{T T}(\pi)}{2 \sigma_{T}(\pi)}=\frac{1}{2} f(\tilde{t}) \sigma_{T T}(\pi)
$$

From (2.6), $\sigma_{T T}(\pi)=0$, and hence $\theta^{\prime}(\tilde{t})=0$.

Differentiating once again (2.11) and evaluating at $T=\tilde{t}$, since $\theta^{\prime}(\tilde{t})=0$ we get

$$
\theta^{\prime \prime}(\tilde{t})=\frac{-\partial^{2} F / \partial T^{2}}{\partial F / \partial \theta}(\pi, \tilde{t})=\frac{-\sigma_{T T T}(\pi)}{3 \sigma_{T}^{\prime}(\pi)}=\frac{1}{3} f(\tilde{t}) \sigma_{T T T}(\pi)
$$

In view of (2.13), the sign of $\theta^{\prime}(T)$, for $T>\tilde{t}$, depends on $\sigma_{T T T}(\pi)$. In order to compute this value, we first have to solve the system of differential equations for $t_{T T T}$ and $\sigma_{T T T}$, which can be obtained as in Lemma 2.1. Straightforwards calculations give

$$
\begin{aligned}
\sigma_{T T T}(\pi)=\frac{\pi}{8 f(\tilde{t})}\{3 K(\tilde{t})(1-f(\tilde{t}))+ \\
\left.3 f(\tilde{t})^{2}\left(\tilde{h} K^{\prime}(\tilde{t})-K^{\prime \prime}(\tilde{t})\right)+5 f(\tilde{t})^{4} K^{\prime}(\tilde{t})^{2}\right\},
\end{aligned}
$$

which finishes the proof. 
We finally state the key result in order to prove the existence of closed embedded unduloid type curves which are stable. We will apply the results appearing in HL (see also $[\mathbf{K}]$ ). Consider a torus of revolution under the conditions of Lemma 2.1] and let $\gamma_{T_{0}}=\gamma\left(\theta, T_{0}, h_{o}\left(T_{0}\right)\right)$ be a closed embedded unduloid type curve, with $T_{0}$ close enough to $\tilde{t}, T_{0}>\tilde{t}$. We know that $\gamma\left(\theta, T, h_{o}(T)\right)$ is a family of closed embedded unduloid type curves, for $T$ close to $T_{0}$. Then we have

Lemma 2.9. ([HL Lemma 2], [K Theorem 1.3]) Assume $\lambda_{1}<0 \leqslant \lambda_{2}$ for the Jacobi operator in $\gamma_{T_{0}}$, with the notation above. Then $\gamma_{T_{0}}$ is stable if and only if

$$
\frac{d h_{o}}{d T} \frac{d a}{d T}<0
$$

at $T=T_{0}$, where $\frac{d a}{d T}$ is the change of area induced by the variation $\gamma\left(\theta, T, h_{o}(T)\right)$.

2.1. Standard tori of revolution. We now focus on standard tori of revolution of Example 1 given by $f(t)=a+r \cos (t / r)$ and $\tilde{t}=\pi r / 2$. We will see that there exist closed embedded unduloid type curves which are stable in some of these surfaces.

Firstly observe that the assumptions of Lemma 2.1] are verified on any standard torus, and so there will exist a family of closed embedded unduloid type curves parametrized by the maximum point of the $t$-coordinate.

We also point out that the condition shown in Lemma 2.8 is satisfied, since the left term in (2.9) is equal to

$$
\frac{5 a^{2}+9 r^{2}}{r^{4}}
$$

which is strictly positive. Therefore the derivative of the period of unduloid type curves with respect to the maximum point will be positive, for $T>\tilde{t}$. This allows to compute the second eigenvalue for the Jacobi operator associated to these curves.

Lemma 2.10. Let $C$ be a closed embedded unduloid type curve in a standard torus of revolution, close enough to $\mathbb{S}^{1} \times\{\tilde{t}\}$. Then the second eigenvalue $\lambda_{2}$ for the Jacobi operator in $C$ is equal to zero.

Proof. From Lemma 2.4 we know that $\lambda_{2} \leqslant 0$. Assume that $\lambda_{2}<0$. Hence, reasonings of this Section give that, for any fundamental piece $C^{\prime}$ of $C, \lambda_{2}^{N}\left(C^{\prime}\right)=\lambda_{2}<0$. This fact yields a contradiction with Lemma 2.7 and so $\lambda_{2}=0$.

Lemma 2.11. Let $M$ be a standard torus of revolution of Example 1 with $r<a<3 r$. Then, there exist closed embedded unduloid type curves which are stable.

Proof. Consider the family $\gamma\left(\theta, T, h_{o}(T)\right)$ of closed embedded unduloid type curves given in Lemma 2.1, with $T>\tilde{t}$ close enough. Fix $C$ one of these curves. By Lemmae 2.4 and 2.10 we can apply Lemma 2.9 to study the stability of $C$.

By differentiating (2.7), it follows that

$$
F_{T}+F_{h} h_{o}^{\prime}=0
$$

and

$$
F_{T T}+2 F_{T h} h_{o}^{\prime}+F_{h h}\left(h_{o}^{\prime}\right)^{2}+F_{h} h_{o}^{\prime \prime}=0
$$

Differentiating once again and evaluating at $T=\tilde{t}$, taking into account that $h_{o}^{\prime}(\tilde{t})=$ 0 and $F_{h}(\tilde{t}, \tilde{h})=\sigma_{h}(\pi)=0$, we obtain

$$
F_{T T T}(\tilde{t}, \tilde{h})+3 F_{T h}(\tilde{t}, \tilde{h}) h_{o}^{\prime \prime}(\tilde{t})=0,
$$


and so

$$
h_{o}^{\prime \prime}(\tilde{t})=\frac{-F_{T T T}(\tilde{t}, \tilde{h})}{3 F_{T h}(\tilde{t}, \tilde{h})}=\frac{-\sigma_{T T T}(\pi)}{3 \sigma_{T h}(\pi)}=\frac{-\sigma_{T T T}(\pi)}{3 \rho / 2},
$$

with $\rho$ as defined in (2.6). Since $M$ is a standard torus of revolution, by (2.14) we conclude that

$$
h_{o}^{\prime \prime}(\tilde{t})=\frac{9 r^{2}+5 a^{2}}{12 a^{3} r^{2}}>0,
$$

so $h_{o}^{\prime}(T)$ is strictly increasing in $T=\tilde{t}$, and then positive for $T>\tilde{t}$.

On the other hand, since the associated vector field induced by the variation $\gamma\left(\theta, T, h_{o}(T)\right)$ is $\left(t_{T}+h_{o}^{\prime} t_{h}\right) \partial_{t}$, we have that the derivative of the area along this deformation is equal to

$$
\int_{0}^{2 \pi} f(T)\left(t_{T}(\theta)+h_{o}^{\prime}(T) t_{h}(\theta)\right) d \theta
$$

which vanishes when evaluated at $T=\tilde{t}$.

Moreover, the second derivative of the area at $T=\tilde{t}$ is given by

$$
\int_{0}^{2 \pi}\left(f^{\prime}(\tilde{t}) t_{T}(\theta)^{2}+f(\tilde{t})\left(t_{T T}(\theta)+h_{o}^{\prime \prime}(\tilde{t}) t_{h}(\theta)\right)\right) d \theta
$$

which equals

$$
\frac{\left(a^{2}-9 r^{2}\right) \pi}{6 r^{2}}<0
$$

since $a<3 r$. Hence, the derivative of the area is strictly decreasing in $T=\tilde{t}$, and so, strictly negative for $T>\tilde{t}$. By applying Lemma 2.9 we conclude that $C$ is stable.

\section{Stable Regions}

In this section we will describe the different stable regions that can appear in our surfaces. As we know which are the stable constant geodesic curvature curves by the previous Section, we will check which combinations of them bound stable regions.

Lemma 3.1. ( $\underline{\mathbf{R}}$ Lemma 1.7]) Consider an annulus $\mathbb{S}^{1} \times\left[t_{1}, t_{2}\right]$ in $M$. Its boundary is stable if and only if each component $\mathbb{S}^{1} \times\left\{t_{i}\right\}$ is stable $(i=1,2)$ and

$$
\frac{K+h^{2}}{L}\left(t_{1}\right)+\frac{K+h^{2}}{L}\left(t_{2}\right) \leqslant 0 .
$$

In the case of a symmetric annulus $\mathbb{S}^{1} \times[-t, t]$, above conditions are equivalent to

$$
\left(K+h^{2}\right)(t) \leqslant 0 .
$$

The above result completely characterizes the stable annuli bounded by parallels. It yields that $\mathbb{S}^{1} \times[t,-t]$ is stable for $t \in\left(-t_{0}, t_{c}\right)$, with $t_{c}$ the supremum of the points in $\left[-t_{0}, 0\right]$ where $K+h^{2}$ (or equivalently $\left(f^{\prime}\right)^{2}-f f^{\prime \prime}$ ) is nonpositive. Moreover, $\mathbb{S}^{1} \times\left[t_{c},-t_{c}\right]$ is stable if and only if $\left(K+h^{2}\right)\left(t_{c}\right)=0$ (recall the possible discontinuities of $K)$. As $h^{\prime}=-\left(K+h^{2}\right)$, it is clear that $h(t)$ is increasing in $\left(-t_{0}, t_{c}\right)$ and decreasing in $\left(t_{c}, 0\right)$. Let $\tilde{t} \in\left[t_{c}, 0\right]$ be the infimum ot the points where $L^{2}\left(K+h^{2}\right) \geqslant 4 \pi^{2}$ (equivalently $\left(f^{\prime}\right)^{2}-f f^{\prime \prime} \geqslant 1$ ). Then, taking into account Lemma 1.8 and Remark 1.6 it follows that for any $t^{\prime} \in\left(t_{c}, \tilde{t}\right), \mathbb{S}^{1} \times\left\{t^{\prime}\right\}$ is stable and there exists a unique $t^{\prime \prime} \in$ $\left(-t_{c}, t_{0}\right)$ satisfying that $\mathbb{S}^{1} \times\left[t^{\prime}, t^{\prime \prime}\right]$ is a nonsymmetric annulus whose boundary has constant geodesic curvature. 
In the light of Lemma 2.2 stable domains bounded by an unduloid type curve and a circle of revolution will appear if all nonsymmetric annuli $\mathbb{S}^{1} \times\left[t^{\prime}, t^{\prime \prime}\right]$, for $t^{\prime} \in\left(t_{c}, \tilde{t}\right)$, are stable, and the first of such domains will arise from the annulus corresponding to $t^{\prime}=\tilde{t}$.

A vertical annulus is a set bounded by two vertical geodesics. The following result proves that the union of vertical annuli is always stable.

Lemma 3.2. Any union of finite disjoint vertical annuli is a stable region.

Proof. Given a vertical geodesic $C$, we shall first prove that its length is less than or equal to the length of any other closed embedded curve $D$, with the same type of homothopy and near enough to $C$.

Let $N$ denote the unit normal vector field to the set of all vertical geodesics, which can be properly extended to $M$. In fact, $N=\frac{1}{f} \partial \theta$. Then, $\operatorname{div}(N)=0$. We can assume without loss of generality that $D$ intersects $C$, consider two consecutive intersection points $p_{1}, p_{2}$, and call $\Sigma$ to one of the domains bounded by the pieces of $C$ and $D$ between $p_{1}$ and $p_{2}$. By applying the Divergence Theorem to $N$ in $\Sigma$, we easily conclude that the length of $C$ is less than or equal to the length of $D$.

Now it is clear that for a vertical annulus $\Omega$, any variation of $\partial \Omega$ preserving the area enclosed will give more perimeter at each instant, so $\partial \Omega$ will be a local minimum for the length while keeping constant the area. Therefore $\Omega$ is stable. Notice that the same argument holds for an arbitrary union of disjoint vertical annuli.

Now we can state our Main Theorem, describing all possible stable regions in our surfaces.

Theorem 3.3. Let $M$ be a rotationally symmetric torus with a horizontal symmetry and with possibly discontinuous, decreasing Gauss curvature from the longest parallel. Then the stable regions in $M$ may be:

i) disks bounded by constant geodesic curvature curves, which are symmetric with respect to the longest parallel, or contained in a region with constant Gauss curvature, and their complements,

ii) annuli symmetric with respect to the shortest parallel, bounded by circles of revolution contained in the region $K+h^{2} \leqslant 0$, and their complements,

iii) nonsymmetric annuli bounded by circles of revolution contained in the region $K+h^{2} \leqslant \frac{4 \pi^{2}}{L^{2}}$ and verifying condition (3.1), and their complements,

iv) unions of vertical annuli bounded by vertical geodesics,

v) annuli bounded by an unduloid type curve satisfying Lemma 2.2 and a circle of revolution contained in $K+h^{2}<0$, and their complements,

vi) unions of a disk and a symmetric annulus with the same geodesic curvature, in the above conditions, and their complements.

Proof. Let $\Omega$ be a stable region in $M$. Then $\partial \Omega$ is an embedded stable curve (not necessarily connected) with constant geodesic curvature with respect to the inner normal. By Theorem 1.4 $\partial \Omega$ will consist of a union of circles of revolution, vertical geodesics, nodoid type curves and unduloid type curves.

First observe that no more than two circles of revolution can appear in $\partial \Omega$, because the geodesic curvatures will not coincide in view of Remark 1.6 
Let $C_{1}, C_{2}$ denote two closed embedded curves with constant geodesic curvature. Assume that the first eigenvalues for the Jacobi operator (1.5) satisfy $\lambda_{1}\left(C_{1}\right) \leqslant 0$ and $\lambda_{1}\left(C_{2}\right)<0$. We will see that $C_{1} \cup C_{2}$ is an unstable curve.

Fix $\phi_{1}$ (resp. $\left.\phi_{2}\right)$ an eigenfunction associated to $\lambda_{1}\left(C_{1}\right)$ (resp. $\lambda_{1}\left(C_{2}\right)$ ). Then we have $J\left(\phi_{1}\right)+\lambda_{1}\left(C_{1}\right) \phi_{1}=0$. Take $\alpha \in \mathbb{R}$ such that

$$
\alpha \int_{C_{1}} \phi_{1}+\int_{C_{2}} \phi_{2}=0
$$

Hence the function

$$
u=\left\{\begin{array}{l}
\alpha \phi_{1}, \quad \text { in } C_{1}, \\
\phi_{2}, \quad \text { in } C_{2}
\end{array}\right.
$$

has mean zero and gives

$$
I(u)=\alpha^{2} \lambda_{1}\left(C_{1}\right) \int_{C_{1}} \phi_{1}^{2}+\lambda_{1}\left(C_{2}\right) \int_{C_{2}} \phi_{2}^{2}<0,
$$

so $C_{1} \cup C_{2}$ is not stable.

We know that the first eigenvalue for the Jacobi operator is negative in unduloid type curves, by Lemma 2.4 and in nodoid type curves, by Remark [2.6 Moreover, for a parallel $\mathbb{S}^{1} \times\{t\}$, it is easy to check that

$$
\lambda_{1}(t)=-\frac{\left(f^{\prime}\right)^{2}-f f^{\prime \prime}}{f^{2}}(t)=-\left(K+h^{2}\right)(t) .
$$

Hence $\lambda_{1}(t)$ is negative if $\left(K+h^{2}\right)(t)>0$, and vanishes if $\left(K+h^{2}\right)(t)=0$. Finally, for any vertical geodesic $C,\left.f\right|_{C}$ is a positive eigenfunction with zero eigenvalue, and so $\lambda_{1}(C)=0$.

In case an unduloid type curve belongs to $\partial \Omega$, it follows from the above arguments that necessarily $\Omega$ is a set bounded by such a curve and a circle of revolution $\mathbb{S}^{1} \times\{t\}$ with positive first eigenvalue, equivalently $\left(K+h^{2}\right)(t)<0$, and then $\Omega$ is a region of type $\mathrm{v})$.

In case a nodoid type curve belongs to $\partial \Omega$, an analogous reasoning will give that $\Omega$ is a region of type i) (by Lemma 1.10) or of type vi).

Assume now that the boundary of $\Omega$ does not contain neither an unduloid type curve nor a nodoid type curve. By Lemma 3.2 any region of type iv) is stable, and if circles of revolution appear in $\partial \Omega$, the remaining possibilities are regions of type ii), by Lemma 3.1 and of type iii), by Lemma 1.8 and the first observation above in the proof.

Remark 3.4. We will now check that sets of type $v$ ), annuli bounded by an unduloid type curve and a circle of revolution, appear as stable regions in some surfaces. Consider a standard torus of revolution of Example 1 with $r<a<3 r$. Denote by $\gamma_{T}$ the unduloid type curve $\gamma\left(\theta, T, h_{o}(T)\right)$, with initial condition $T$ close to $\tilde{t}=\pi r / 2$. By Lemma 2.11 these curves are stable. Let $\Omega_{T}$ be the annulus bounded by $\gamma_{T}$, and the corresponding circle of revolution $\mathbb{S}^{1} \times\{t(T)\}$, with positive first eigenvalue (that is, contained in $K+h^{2}<0$ ) and with the same geodesic curvature with respect to the inner normal. Let us prove that $\Omega_{T}$ is stable.

Consider $u$ any mean zero function defined on $\partial \Omega_{T}$, normalized so that $\int_{\partial \Omega} u^{2}=1$, and let $u_{1}, u_{2}$ be the restrictions of $u$ to $\gamma_{T}$ and $\mathbb{S}^{1} \times\{t(T)\}$, respectively. It is possible to express $u_{i}=c_{i}+v_{i}$, with $c_{i}$ a real constant and $v_{i}$ a mean zero function, for $i=1,2$. 
We have

$$
\begin{aligned}
& I(u)=I\left(u_{1}\right)+I\left(u_{2}\right)= \\
& -c_{1}^{2} \int_{\gamma_{T}}\left(K+h^{2}\right)-2 c_{1} \int_{\gamma_{T}} v_{1}\left(K+h^{2}\right)-c_{2}^{2} L(t(T))\left(K+h^{2}\right)(t(T))+ \\
& \quad I\left(v_{1}, v_{1}\right)+I\left(v_{2}, v_{2}\right) .
\end{aligned}
$$

Observe that $I\left(v_{i}, v_{i}\right) \geqslant 0$ by the stability of each boundary curve. Moreover, the mean zero condition on $u$ gives $c_{1} L\left(\gamma_{T}\right)=-c_{2} L(t(T))$, where $L\left(\gamma_{T}\right)$ denotes the lenght of $\gamma_{T}$, and so

$$
\begin{aligned}
I(u) \geqslant & -c_{1}^{2} L\left(\gamma_{T}\right)^{2}\left(\frac{\int_{\gamma_{T}}\left(K+h^{2}\right)}{L\left(\gamma_{T}\right)^{2}}+\frac{\left(K+h^{2}\right)(t(T))}{L(t(T))}\right)- \\
& 2 c_{1} \int_{\gamma_{T}} v_{1}\left(K+h^{2}\right) .
\end{aligned}
$$

When $T$ is close to $\tilde{t}$, it follows that $\gamma_{T}$ is close to $\mathbb{S}^{1} \times\{\tilde{t}\}$ and, consequently, $\Omega_{T}$ is close to the nonsymmetric annulus associated to $\tilde{t}$, namely $\mathbb{S}^{1} \times\left[t^{\prime}, \tilde{t}\right]$. Since this annulus satisfies strictly the stability condition (3.1), we conclude that the first summand in (3.2) is positive for $T$ close enough to $\tilde{t}$.

On the other hand, denoting by $v_{1}^{+}=\max \left\{v_{1}, 0\right\}$ and $v_{1}^{-}=-\min \left\{v_{1}, 0\right\}$ we have that $v_{1}=v_{1}^{+}-v_{1}^{-}$, and so

$$
\begin{aligned}
\int_{\gamma_{T}} v_{1}\left(K+h^{2}\right)= & \int_{\gamma_{T}} v_{1} K=\int_{\gamma_{T}} v_{1}^{+} K-\int_{\gamma_{T}} v_{1}^{-} K \leqslant \\
& K\left(s_{1}\right) \int_{\gamma_{T}} v_{1}^{+}-K\left(s_{2}\right) \int_{\gamma_{T}} v_{1}^{-},
\end{aligned}
$$

where $K\left(s_{1}\right)=\max _{\gamma_{T}} K$ and $K\left(s_{2}\right)=\min _{\gamma_{T}} K$. Since $\gamma_{T}$ lies in a narrow band around $\mathbb{S}^{1} \times\{\tilde{t}\}$, we have $K\left(s_{1}\right)-K\left(s_{2}\right)=\varepsilon_{T}$, so that $K\left(s_{1}\right)=K\left(s_{2}\right)+\varepsilon_{T}$ and so

$$
\int_{\gamma_{T}} v_{1}\left(K+h^{2}\right) \leqslant \varepsilon_{T} \int_{\gamma_{T}} v_{1}^{+}
$$

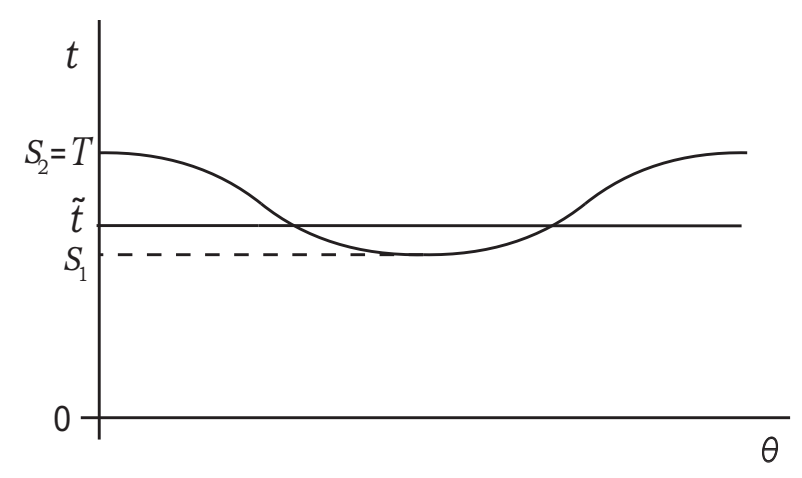

Figure 4. The unduloid type curve $\gamma_{T}$ and the circle of revolution $\mathbb{S}^{1} \times\{\tilde{t}\}$

Hence

$$
\left|\int_{\gamma_{T}} v_{1}\left(K+h^{2}\right)\right| \leqslant \varepsilon_{T}\left|\int_{\gamma_{T}} v_{1}^{+}\right| \leqslant \varepsilon_{T}\left(\int_{\gamma_{T}}\left(v_{1}^{+}\right)^{2}\right)^{\frac{1}{2}} L\left(\gamma_{T}\right)^{\frac{1}{2}} .
$$


Since

we obtain

$$
\int_{\gamma_{T}}\left(v_{1}^{+}\right)^{2} \leqslant \int_{\gamma_{T}} v_{1}^{2} \leqslant \int_{\gamma_{T}} u_{1}^{2}=\int_{\gamma_{T}} u^{2} \leqslant 1
$$

$$
\left|\int_{\gamma_{T}} v_{1}\left(K+h^{2}\right)\right| \leqslant \varepsilon_{T} L\left(\gamma_{T}\right)^{\frac{1}{2}}
$$

so that the second summand in (3.2) is negligible, when $T$ is close enough to $\tilde{t}$. We conclude that $I(u)$ is positive and hence $\Omega_{T}$ is stable, for $T$ close to $\tilde{t}$.

Remark 3.5. We will now show that sets of type vi), unions of a disk and a symmetric annulus, actually occur as stable regions in some surfaces. Let $\widetilde{M}$ be one of the surfaces of the family described in Example 2$]$ obtained from a sphere and two hyperbolic annuli.

Given a disk $D \subset \widetilde{M}$, bounded by a closed embedded nodoid type curve, and a symmetric annulus $B \subset \widetilde{M}$ with $h(\partial D)=h(\partial B)=h$ with respect to the inner normal, following lemma states the stability condition for $\Omega=D \cup B$.

Lemma 3.6. In the above conditions, $\Omega=D \cup B \subset \widetilde{M}$ is stable if and only if $D$ and $B$ are stable sets, and

$$
\frac{\left(a^{2}+h^{2}\right)^{3 / 2}}{2 \pi}-\frac{\left(b^{2}-h^{2}\right)^{3 / 2}}{4 \pi b c} \leqslant 0,
$$

where $a^{2}$ and $-b^{2}$ are respectively the Gauss curvatures of the initial sphere and hyperbolic annuli, and $2 \pi c$ is the minimum length of a circle of revolution contained in the hyperbolic piece.

Proof. Suppose first that $D$ and $B$ are stable and (3.3) holds. Let $u$ be a mean zero function defined on $\partial \Omega$, and name $u_{1}, u_{2}$ the restrictions of $u$ to $\partial D, \partial B$, respectively. It is possible to express $u_{i}=c_{i}+v_{i}$, with $c_{1}, c_{2}$ real constants and $v_{1}, v_{2}$ mean zero functions on $\partial D$ and $\partial B$. These decompositions easily allows to check that

$$
\begin{aligned}
I(u) & =I\left(u_{1}\right)+I\left(u_{2}\right) \geqslant I\left(c_{1}\right)+I\left(c_{2}\right) \\
& =-L(\partial D) c_{1}^{2}\left(a^{2}+h^{2}\right)-L(\partial B) c_{2}^{2}\left(-b^{2}+h^{2}\right),
\end{aligned}
$$

since $v_{i}$ has mean zero and $D$ and $B$ are stable, and where $L$ denotes the length. The mean zero value condition of $u$ gives $c_{1} L(\partial D)=-c_{2} L(\partial B)$, and then

$$
I(u) \geqslant-L^{2}(\partial D) c_{1}^{2}\left(\frac{a^{2}+h^{2}}{L(\partial D)}+\frac{-b^{2}+h^{2}}{L(\partial B)}\right) .
$$

By Lemma 3.1 we know that $B=\mathbb{S}^{1} \times[-t, t]$ will be contained in the hyperbolic piece since it is stable. Then

$$
L(\partial B)=2 L(t)=4 \pi c \cosh (d-b t)=4 \pi b c\left(b^{2}-h^{2}\right)^{-1 / 2},
$$

where in the last equality we have used that $h(t)=h$. Straightforward calculations give that $L(\partial D)=2 \pi\left(a^{2}+h^{2}\right)^{-1 / 2}$. Therefore

$$
I(u) \geqslant-L^{2}(\partial D) c_{1}^{2}\left(\frac{\left(a^{2}+h^{2}\right)^{3 / 2}}{2 \pi}-\frac{\left(b^{2}-h^{2}\right)^{3 / 2}}{4 \pi b c}\right) .
$$

As we are assuming that (3.3) is verified, then (3.4) is nonnegative, and so $\Omega$ is stable.

Assume now that $\Omega$ is a stable region. Then clearly $D$ and $B$ are also stable. Consider the mean zero function

$$
u=\left\{\begin{array}{l}
L(\partial D), \quad \partial B \\
-L(\partial B), \quad \partial D .
\end{array}\right.
$$


Using this function in the index form, we will have $I(u) \geqslant 0$ by the stability of $\Omega$, which trivially gives (3.3).

The above lemma gives us the existence of surfaces where sets of type vi) are stable. For instance, consider the surface $\widetilde{M}$ given by $a=1, t^{*}=\pi / 6$, with $b=0.578$ and $c=0.0410512$. Then it can be checked that, for geodesic curvature $h=0.4$, there exists a stable union of a disk and a symmetric annulus.

\section{ISOPERIMETRIC REGIONS}

Since we are studying compact surfaces, well-known results from geometric measure theory $\mathbf{M}$ ensure the existence of isoperimetric solutions for any value of the area. Moreover, as any isoperimetric region is stable, the candidates are given by Theorem 3.3. We first discard sets of tipe iv) composed by several vertical annuli.

Lemma 4.1. The union of two or more vertical annuli is not an isoperimetric region.

Proof. Simply rotate a vertical annulus until meeting another one and, after eliminating the duplicated vertical geodesic, we get a less-perimeter region enclosing the same area.

The following lemma shows that when the boundary of an isoperimetric region is an unduloid type curve and a circle of revolution, some restrictions appear. Call lower half of $M$ to $\mathbb{S}^{1} \times\left[-t_{0}, 0\right]$ and upper half to $\mathbb{S}^{1} \times\left[0, t_{0}\right]$.

Lemma 4.2. Let $\Omega$ be an isoperimetric region in the warped product $\mathbb{S}^{1} \times\left[-t_{0}, t_{0}\right]$ bounded by an unduloid type curve $C$ and a circle of revolution $\mathbb{S}^{1} \times\{t\}$. Then

i) $C$ and $\mathbb{S}^{1} \times\{t\}$ are not contained in the same half.

ii) $C$ does not intersect $\mathbb{S}^{1} \times\{-t\}$.

Proof. Without loss of generality we can assume that $t \in\left[-t_{0}, 0\right]$. Since $\Omega$ has to be stable, by Theorem 3.3 we have $\left(K+h^{2}\right)(t)<0$. Consequently, $t \in\left[-t_{0}, t_{c}\right]$.

i) Suppose both curves lie in the half $\mathbb{S}^{1} \times\left[-t_{0}, 0\right]$. Call $t_{m}, t_{M}$ the minimun and the maximum of $\left.t\right|_{C}$, respectively. If $t=-t_{0}$, it can be checked by using (1.3) that $f\left(t_{m}\right)=f\left(t_{M}\right)$, and then $C$ intersects symmetrically $\mathbb{S}^{1} \times\{0\}$. This contradicts the fact that $C$ is contained in the lower half. Hence $t \neq-t_{0}$. Since $C$ has to intersect $\left(f^{\prime}\right)^{2}-f f^{\prime \prime}=1$, it will be necessarily contained in $\mathbb{S}^{1} \times[t, 0]$. But then we can construct a new set enclosing the same area with strictly less perimeter: consider a parallel $\mathbb{S}^{1} \times\left\{t^{*}\right\}$ intersecting $C$, with $t^{*}$ close to $t_{M}$, and replace the piece of $C$ above the parallel by the corresponding segment of parallel. Also replace $\mathbb{S} \times\{t\}$ by $\mathbb{S} \times\{\bar{t}\}$ with $\bar{t}<t$. There exist appropriate $t^{*}$ and $\bar{t}$ for which the new set encloses the same area and it can be checked that it has less perimeter, which is contradictory since $\Omega$ is isoperimetric.

ii) Suppose $C$ intersects $\mathbb{S}^{1} \times\{-t\}$. Replace the piece of $C$ above the parallel $\mathbb{S}^{1} \times\{-t\}$ by the corresponding segment of the parallel. By reflecting the replaced piece with respect to $\mathbb{S}^{1} \times\{0\}$ and removing a segment of $\mathbb{S}^{1} \times\{t\}$, we obtain a new set with the same perimeter and enclosing the same area that $\Omega$, but with no regular boundary, which is a contradiction.

Remark 4.3. Assume that a disk with constant geodesic boundary, and contained in a region with constant Gauss curvature, is a component of an isoperimetric region. 
Then, by using a comparison argument ( $\mathbf{R}$, Lemma 2.7), it can be checked that such a constant is precisely the maximum of the Gauss curvature.

4.1. Isoperimetric regions in standard tori. We will now focus on standard tori described in Example 1. Denote by $\beta$ the total area of a given torus. Fixing the value of $a$, we have studied the isoperimetric solutions for the different values of $r$.

Theorem 4.4. Consider a standard torus, parametrized by $r$ and $a$, with $r \leqslant a / 2$. Then the isoperimetric regions are disks with constant geodesic curvature, and vertical annuli, and their complements.

Proof. Since $r \leqslant a / 2$, it is easy to see that the length of any circle of revolution, and therefore of any unduloid type curve, is greater than or equal to the length of a vertical geodesic. This fact reduces the candidates to disks and vertical annuli, which actually appear as isoperimetric.

When $r>a / 2$ we have observed, with numerical computations, different behaviors as $r$ increases. For values of $r$ close to $a / 2$, it happens as in Theorem 4.4 there are also values of $r$ for which disks (or complements of them) are solutions for any quantity of the area; then, there exists an interval for $r$ where the isoperimetric regions are disks, symmetric annuli afterwards, and finally disks for values of the area near $\beta / 2$; and for $r$ close enough to $a$, we get disks, symmetric annuli, nonsymmetric annuli and disks again as solutions.

The perimeter of the isoperimetric candidates, as a function of the area enclosed, is depicted in two different standard tori of revolution in Figure 5 In each graph, the curve starting from the origin corresponds to the perimeter of disks, and the above one not touching the vertical axis corresponds to the perimeter of unions of a disk and a symemtric annulus. In the first graph, we observe that the perimeter of disks is less than any other set, for any quantity of area. However, in the second one, symmetric and nonsymmetric annuli are also solutions (the black dots show the transition between symmetric and nonsymetric annuli, and between nonsymmetric annuli and sets of type v)).

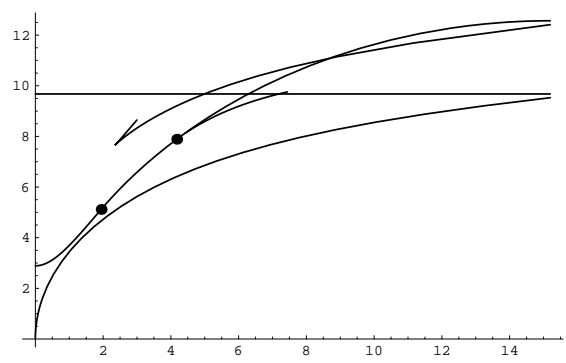

(a) $r=0.77 a$

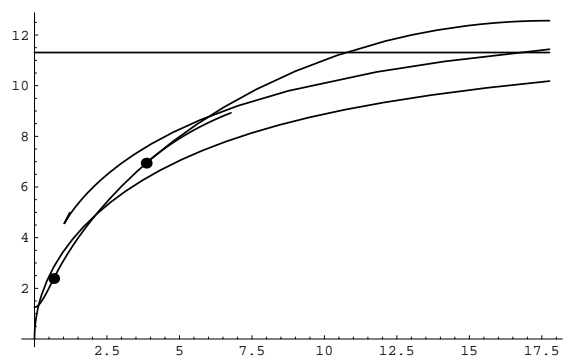

(b) $r=0.9 a$

FiguRE 5. Graphs showing the perimeter of each candidate in two different standard tori

Remark 4.5. We have not found any stardard torus where a domain bounded by an unduloid type curve and a circle of revolution is an isoperimetric region. 
Remark 4.6. We have observed numerically that for any standard torus, the isoperimetric region for area $\beta / 2$ is either a disk with constant geodesic boundary, or a vertical annulus. We will see that this fact does not hold in others surfaces.

4.2. Isoperimetric regions in $\widetilde{M}$. Consider now the surfaces $\widetilde{M}$ introduced in Example 2 and call as above $\beta$ the total area of the surface. We have studied carefully the isoperimetric problem in this case, specially when vertical annuli have too much perimeter (that is, when hyperbolic annuli are long enough), and hence they are not isoperimetric solutions. In this setting, we have seen that isoperimetric regions are disks bounded by constant geodesic curvature curves for small areas (entirely contained in the spherical piece, where the Gauss curvature achieves constantly its maximum), and afterwards symmetric annuli (with its boundary contained in the hyperbolic piece); or disks, all symmetric annuli which are stable, and finally nonsymmetric annuli for quantities of area near $\beta / 2$ (with a circle of revolution of its boundary in the spherical piece, and another one in the hyperbolic part).

Annuli bounded by an unduloid type curve and a circle of revolution also appear as isoperimetric regions in these surfaces. Consider a nonsymmetric annulus which is an isoperimetric region in $\widetilde{M}$. By rotating slightly the spherical piece, keeping unchanged the hyperbolic annuli, we will obtain another isoperimetric region, since area and perimeter are preserved. This new region is now bounded by an unduloid type curve (contained in the spherical part) and a circle of revolution (in the hyperbolic one).

Then we have

Theorem 4.7. Let $\widetilde{M}$ be one of the surfaces described in Example 2 obtained from a sphere and a hyperbolic annulus. Then the isoperimetric regions may be disks with constant geodesic boundary, symmetric or nonsymmetric annuli, annuli bounded by an unduloid type curve and a circle of revolution, unions of a disk and a symmetric annulus, vertical annuli or the complement of one of these sets.

Remark 4.8. In PR Prop. 3.4.] it is proved that in high dimensions, there are isoperimetric domains bounded by hypersurfaces of revolution generated by unduloid type curves. Up to our knowledge, this kind of curves had not appear as part of the boundary of an isoperimetric solution in any surface. The above result shows that this fact may occur.

Remark 4.9. Note that for a surface $\widetilde{M}$ of Example 2 symmetric annuli, nonsymmetric annuli and annuli bounded by an unduloid type curve and a circle of revolution may be isoperimetric solutions for area $\beta / 2$.

Remark 4.10. Although a set consisting of a disk and a symmetric annulus can be stable (Lemma 3.6), we have not found any surface where such a region is isoperimetric. For instance, this kind of sets does not appear in most of the surfaces $\widetilde{M}$, since the constant geodesic curvature condition is hardly verified. However, it seems that only a length comparison argument might discard them as isoperimetric.

\section{REFERENCES}

[BB] L. Barbosa, P. Bérard, Eigenvalue and "twisted" eigenvalue problems, applications to CMC surfaces, J. Math. Pures Appl. 9 (2000), no. 5, 427-450. MR 2001f:58064

[BGS] J. L. M. Barbosa, J. M. Gomes and A. M. Silveira, Foliation of 3-dimensional space forms by surfaces with constant mean curvature, Bol. Soc. Brasil. Mat. 18 (1987), no. 2, 1-12. MR 90j:53054

[BP] C. Bavard and P. Pansu, Sur le volume minimal de $\mathbf{R}^{2}$, Ann. Sci. École Norm. Sup. (4) 19 (1986), no. 4, 479-490. MR 88b:53048 
[BC] I. Benjamini and J. Cao, A new isoperimetric comparison theorem for surfaces of variable curvature, Duke Math. J. 85 (1996), 359-396. MR 97m:58046

[Ch] I. Chavel, Eigenvalues in Riemannian Geometry, Academic Press, 1984. MR 86g:58140

[CL] E.A. Coddington and N. Levinson, Theory of Ordinary Differential Equations, McGrawHill, New York, 1955. MR 16,1022b

[CHLL] J. Corneli, P. Holt, G. Lee, N. Leger, E. Schoenfeld and B. Steinhurst, The double bubble problem on the flat two-torus, Trans. Amer. Math. Soc. 356 (2004), no. 9, 3769-3820. MR 2005b:53011

[H] H. Howards, Soap bubbles on surfaces, undergraduate thesis, Williams College, 1992.

[HHM1] H. Howards, M. Hutchings and F. Morgan, The isoperimetric problem on surfaces, Amer. Math. Monthly 106 (1999), no. 5, 430-439. MR 2000i:52027

[HHM2] H. Howards, M. Hutchings and F. Morgan, The isoperimetric problem on surfaces of revolution of decreasing Gauss curvature, Trans. Amer. Math. Soc. 352 (2000), no. 11, 4889-4909. MR 2001b:58024

[HL] W. H. Huang, C. C. Lin, Negatively curved sets on surfaces of constant mean curvature in $\mathbb{R}^{3}$ are large, Arch. Rational Mech. Anal. 141 (1998), no. 2, 105-116. MR 99j:58049

[K] M. Koiso, Deformation and stability of surfaces with constant mean curvature, Tohoku Math. J. 54 (2002), 145-159. MR 2003j:58021

[M] F. Morgan, Geometric Measure Theory: a Beginner's Guide, third ed., Academic Press Inc, 2000. MR 2001j:49001

[Mi] J. Milnor, Morse theory, Princeton Univ. Press, Princeton, N.J., 1963. MR 29:634

[O] R. Osserman, Bonnesen-style isoperimetric inequalities, Amer. Math. Monthly 86 (1979), no. 1, 1-29. MR 80h:52013

[P] P. Pansu, Sur la régularité du profil isopérimétrique des surfaces riemanniennes compactes, Ann. Inst. Fourier (Grenoble) 48 (1998), no. 1, 247-264. MR 99i:53035

[PR] R. H. L. Pedrosa, M. Ritoré, Isoperimetric domains in the Riemannian product of a circle with a simply connected space form and applications to free boundary problems, Indiana Univ. Math. J. 48 (1999), no. 4, 1357-1394. MR 2001k:53120

[R] M. Ritoré, Constant geodesic curvature curves and isoperimetric domains in rotationally symmetric surfaces, Comm. Anal. Geom. 9 (2001), no. 5, 1093-1138. MR 2003a:53018

[S] E. Schmidt, Über eine neue Methode zur Behandlung einer Klasse isoperimetrischer Aufgaben im Grossen, Math. Z. 47 (1942), 489-642. MR 7 (1946)

[T] P. Topping, The isoperimetric inequality on a surface, Manuscripta Math. 100 (1999), no. 1, 23-33. MR 2000i:53107

Departamento de Matemáticas, Campus de Ponferrada, Universidad de León, E-24400 LEÓN (EsPAÑA)

E-mail address: antonioc@unileon.es 